EPJ Web of Conferences 19, 08006 (2012)

DOI: $10.1051 /$ epjconf/20121908006

(C) Owned by the authors, published by EDP Sciences, 2012

\title{
The interplay between the young stellar super cluster Westerlund 1, and the surrounding interstellar medium
}

\author{
Y.D. Mayya ${ }^{1, a}$, A. Luna ${ }^{1}$, L. Carrasco ${ }^{1}$ and L. Bronfman ${ }^{2}$ \\ ${ }^{1}$ INAOE, Mexico \\ ${ }^{2}$ Univ. de Chile, Chile
}

\begin{abstract}
We analyze the multi-band (CO, HI and Spitzer maps), large-scale (150 pc) gaseous structure around Westerlund 1, the most massive known superstar cluster in the Milky Way, with the intention of exploring the effect of feedback from massive stars in this young (age $<5 \mathrm{Myr}$ ) cluster on the surrounding interstellar medium. We find no traces of the parental molecular cloud in the immediate vicinity of the cluster, instead this volume is partially filled by HI gas. On the other hand, there are two giant molecular clouds, both moving away from the cluster at $5-10 \mathrm{~km} \mathrm{~s}^{-1}$, at distances of around $50-150 \mathrm{pc}$. There are several ultra-compact HII regions associated with these giant molecular clouds. All these events suggest that the cluster has played an important role in re-structuring the ISM, in the form of ejecting the molecular gas, as well as triggering secondary star formation.
\end{abstract}

\section{INTRODUCTION}

Westerlund 1 is the most massive compact young stellar cluster in the Milky Way, with an estimated stellar mass of $\sim 10^{5} M \odot$, size of $\sim 1 \mathrm{pc}$ and age of $5 \mathrm{Myr}$ ([8]). The cluster stars have a radial velocity of $-55 \mathrm{~km} \mathrm{~s}^{-1}$, which associates it to the Scutum-Crux (SCX) arm, at a distance of $4.3 \mathrm{kpc}$ ([5], [6]). In this study, we analyze the large scale $(150 \mathrm{pc})$ gas environment of this cluster, in order to understand the cluster-interstellar medium feedback processes such as the fate of the parental molecular cloud, secondary star formation in the surrounding clouds etc.

\section{LARGE-SCALE GASEOUS ENVIROMENT OF WESTERLUND 1}

We explore the molecular environment of Westerlund 1 using the Columbia-Calan ${ }^{12} \mathrm{CO}$ survey data ([1]) which has a beam of $8.8^{\prime}$. We analysed the molecular data cube of $\delta b \times \delta l=2^{\circ} \times 3^{\circ}$ centered on the cluster $(150 \mathrm{pc} \times 225 \mathrm{pc}$ at the distance of Westerlund 1) between radial velocities of -35 and $-68 \mathrm{~km} \mathrm{~s}^{-1}$. A map of the $\mathrm{CO}$ emission is shown overlaid on the $24 \mu \mathrm{m}$ image in Figure 1 . Most of the molecular gas in the mapped region is contained in two giant molecular clouds (GMCs) each one of mass $\sim 10^{6} M \odot$. These clouds are situated at distances $50 \mathrm{pc}$ east and $150 \mathrm{pc}$ northwest, with the former cloud (named $R$ ) redshifted by $10 \mathrm{~km} \mathrm{~s}^{-1}$ and the latter one blueshifted by $5 \mathrm{~km} \mathrm{~s}^{-1}$ (named $B$ ) with respect to the systemic velocity of the cluster. There are two more moving clouds of lesser mass (denoted $R^{\prime}$ and $B^{\prime}$ ) at distances larger than $50 \mathrm{pc}$ from the cluster center. There is very little molecular gas (mass $\sim 4 \times 10^{3} \mathrm{M} \odot$ ) at the rest velocity of the cluster in a diameter of $\sim 50 \mathrm{pc}$ (the molecular masses are estimated using $\mathrm{N}(\mathrm{H} 2) / \mathrm{W}^{12} \mathrm{CO}=1.56 \times 10^{20} \mathrm{~cm}^{-2} \mathrm{~K}^{-1} \mathrm{~km} \mathrm{~s}^{-1}$; [3]). We also analyzed the $\mathrm{HI}$ and the 20 -cm radio continuum maps using the data from the Milky Way disk survey

\footnotetext{
ae-mail: ydm@inaoep.mx
}

This is an Open Access article distributed under the terms of the Creative Commons Attribution-Noncommercial License 3.0, which permits unrestricted use, distribution, and reproduction in any noncommercial medium, provided the original work is properly cited. 


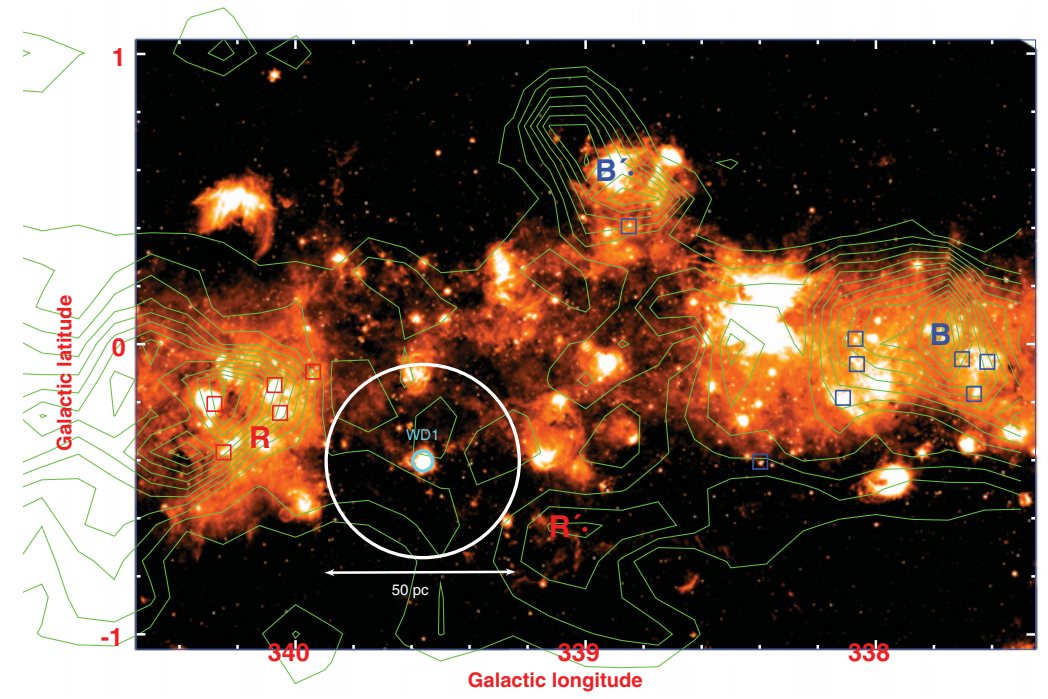

Figure 1. Contours of ${ }^{12} \mathrm{CO}$ emission, integrated between velocities of -35 and $-68 \mathrm{~km} \mathrm{~s}^{-1}$, superposed on the $24 \mu \mathrm{m}$ image from Spitzer. The position of the cluster Westerlund 1 is shown by the blue circle. Most of the molecular gas is located at the positions denoted by $R, B, R^{\prime}$ and $B^{\prime}$ and are receeding the cluster by $5-10 \mathrm{~km} \mathrm{~s}^{-1}$. Note that there is hardly any molecular gas in the immediate vicinity of the cluster (white circle). Square symbols denote embedded young stellar objects as traced by the CS(2-1) emission line [2].

[7], and found that the immediate vicinity of the cluster is almost empty of gas in atomic or ionized form, with the mass enclosed within a diameter of $50 \mathrm{pc}$ of the cluster being much less than the mass of the gas that a young cluster of $\sim 10^{5} \mathrm{M} \odot$ is capable of ionizing $\left(\sim 10^{5} \mathrm{M} \odot\right)$ in case the region is ionization bounded. Thus, the residual material of the cloud that formed the cluster is not present within a radius of $25 \mathrm{pc}$.

Given that Westerlund 1 is located within half a degree from the mid-plane of the galactic disk, the absence of gas in any form at significant levels in the immediate vicinity of this cluster indicates either that the cluster is formed with $100 \%$ efficiency or that the residual gas has been expelled to larger distances. The GMCs moving radially away from the cluster might be parts of the massive parental cloud. The farthest of the clouds $(B)$ is at a distance of $150 \mathrm{pc}$ from the cluster. Given an upper limit to the cluster age of $5 \mathrm{Myr}$, the cloud should have been receeding at velocities greater than $30 \mathrm{~km} \mathrm{~s}^{-1}$ to reach its present location. This would require kinetic energies of $\sim 10^{52} \mathrm{erg} \mathrm{s}^{-1}$. A cluster of $10^{5} M \odot$ deposits an order of magnitude more mechanical energy in the first $5 \mathrm{Myr}$ ([4]). Thus, it is possible that the GMCs $B$ and $R$ were once part of the molecular cloud that formed the cluster Westerlund 1. These clouds have several young stellar objects as traced by the $24 \mu \mathrm{m}$ emission, with associated CS emission at the expected velocity of the clouds ([2]). These objects are still in their embedded phase, and hence star-formation in these clouds started after the formation of Westerlund 1. All these events suggest that the cluster has played an important role in re-structuring the ISM, in the form of ejecting the molecular gas, as well as triggering secondary star formation.

\section{References}

[1] Bronfman, L., Alvarez, H., Cohen, R., \& Thaddeus, P. 1989, ApJS, 71, 481

[2] Bronfman, L. Nyman, L., \& May, J. 1996, A\&A, 115, 81

[3] Hunter, S., Bertsch, D., Catelli, J., et al. 1997, ApJ, 481, 205 
Assembling the Puzzle of the Milky Way

[4] Leitherer, C. et al. 1999, ApJS, 123, 3

[5] Luna, A., Mayya, Y.D., Carrasco, L. \& Bronfman, L. 2010, ApJ, 713, L49

[6] Luna, A., Mayya, Y.D., Carrasco, L. \& Bronfman, L. 2011, RevMexAA (Conf Series), 40, 173

[7] McClure-Griffiths, N. M., et al. 2005, ApJS, 158, 178

[8] Negueruela, I., Clark, J.S., \& Ritchie, B.W. 2010, A\&A, 516, A78 